\title{
Modular Robot Based on 3 Rotational DoF Modules
}

\author{
Ariadna Yerpes, Jose Baca, Juan A. Escalera, Member, IEEE, \\ Manuel Ferre, Member, IEEE, Rafael Aracil, Member, IEEE
}

\begin{abstract}
- this paper shows the advantages of having a modular system with 3-DoF spherical actuator in the base module to perform tasks that require displacement and object manipulation. Having 3-DoF actuator improves the complexity of coordination patterns and control algorithms of the modular system more relevantly as compared to having only 1 or $2 \mathrm{DoF}$ actuator in the module. Nevertheless, modules with actuators of only 1 or $2 \mathrm{DoF}$ require more modules to be assembled together in order to achieve complex tasks. Experiments performed with RobMAT modular system proves that a $3 \mathrm{DoF}$ actuator in the module is better, because tasks such as displacement, obstacle climbing and object manipulation, can be efficiently carried out with systems of 2 modules, 4 modules and 6 (maximum) modules connected together.
\end{abstract}

\section{INTRODUCTION}

Since the development of the first modular robots [1] the main idea behind its research work is to develop systems, wherein the whole is greater than the sum of its parts, i.e. wherein performance of the complete modular robot is better that the sum of its module's performance. One of the first modular robots developed, CEBOT [2], already followed this philosophy that has been inherited by the systems developed afterwards [3] [4] [5] [6], including the system used for this experimentation, RobMAT. However, the way this idea has been interpreted and developed, varies depending on the modular robot being considered. Due to such, homogenous (unit-modular) or heterogeneous (nmodular) systems, lattice, chain, or mobile geometries and systems with mechanical coupling or without coupling [7] [8] can be found. The aim of this paper is to show the advantages of having a module with 3-DoF spherical actuator to perform tasks such as, displacement and object manipulation. Using this kind of actuator means an increase in complexity of control algorithms and coordination of modules, since it requires more variables to control than just $1 \mathrm{DoF}$ or $2 \mathrm{DoF}$ actuator. However, as most modular systems with these motors are able to either displace or manipulate, few are able

Manuscript received February 21, 2008. This work was supported in part by the Spanish Ministerio de Educacin, under Grant DPI 2003-00759 and DPI-2006-06493.

A. Yerpes is with the Group of Intelligent Machines, Universidad Politcnica de Madrid, Madrid, Spain ayerpes @etsii.upm.es

J. Baca is with the Group of Intelligent Machines , Universidad Politcnica de Madrid, Madrid, Spain jbaca@etsii.upm.es

Andoni Crespo is with the Group of Intelligent Machines, Universidad Politcnica de Madrid, Madrid, Spain andonicrespoeyahoo.es

J. A. Escalera is with the Group of Intelligent Machines , Universidad Politcnica de Madrid, Madrid, Spain jescalera@etsii.upm.es

M. Ferre is with the Group of Intelligent Machines, Universidad Politcnica de Madrid, Madrid, Spain m. ferre@upm.es

R. Aracil is with the Group of Intelligent Machines, Universidad Politcnica de Madrid, Madrid, Spain Rafael. aracil@upm.es to carry both tasks at the same time without a large number of module reconfigurations. This paper shows the complexity of connecting a 3-DoF motor in the module is highly justified by the advantages that can be derived. Advantages such as having a smaller number of modules connected together, with no reconfiguration or with only one reconfiguration, in performing object manipulation and displacement tasks. Experiments carried out with RobMAT's modular system [9], are described as follows; the first part explains displacement, followed by object manipulation and field work. Conclusions are written at the end of the paper.

\section{DESCRIPTION OF THE SYSTEM USED IN THE EXPERIMENT}

RobMAT's system architecture is divided into modules, molecules and colonies. A MODULE is the base component of the system and it has movement and communication capacity. A MOLECULE is an autonomous entity made out of $n$-modules connected together $(n>1)$. The molecule holds higher capabilities of movement, communication and manipulation than the module. Various molecules cooperating together to fulfill a task is defined as COLONY. The system has three communication channels. These communication channels are the Intra-Module Communication, the InterModules Communication and the Video Communication.

The first type is in charge of the communication between modules belonging to the same molecule and it uses a CAN bus [11]. The Inter-Modules Communication is in charge of communication between molecules or between a molecule

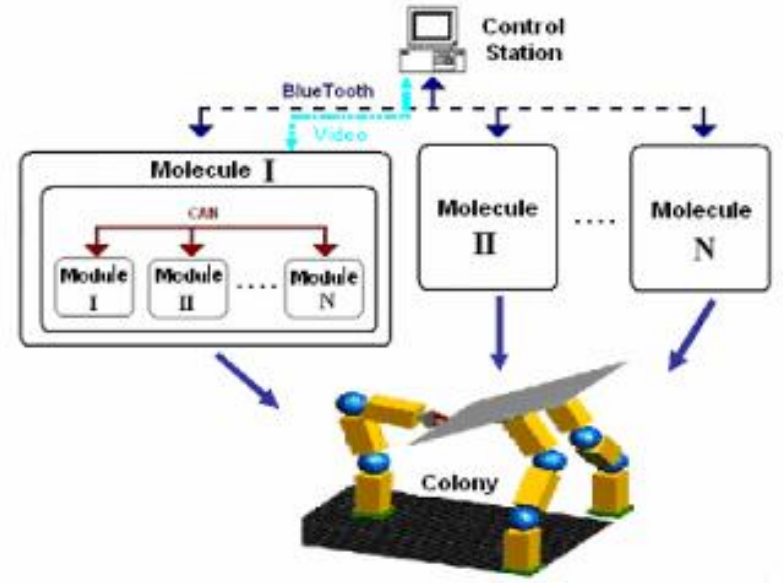

Fig. 1. Components of RobMAT system architecture and molecules cooperating to perform a manipulation task 

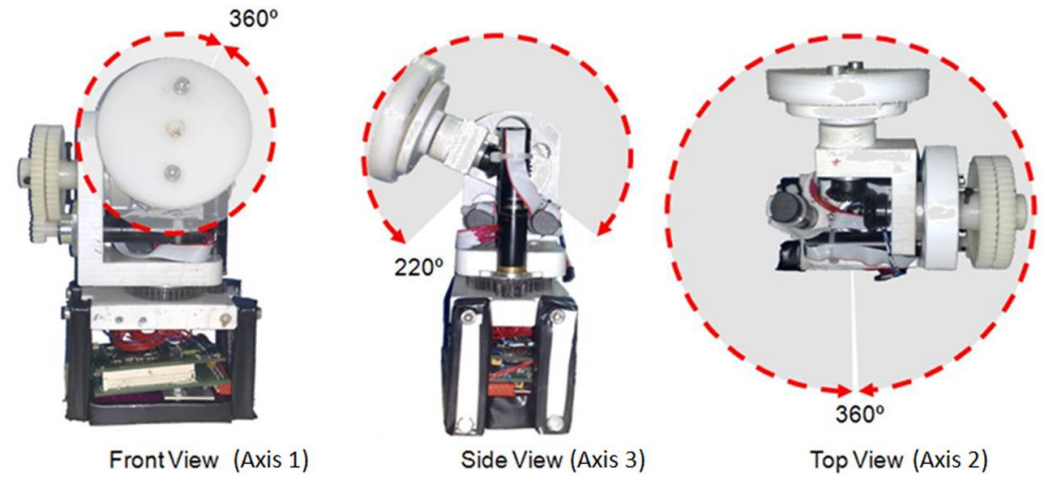

Fig. 2. Working area of each degree of freedom of the 3DoF actuators in the modules

and the control station and it is based on Bluetooth. Finally, the Video Communication is used to send radio frequency video signals from the two mini cameras that can be attached to one of the molecules and that would be used to control the system in a remote working environment using teleoperation [14]. Figure 1 shows a simulation of six modules collaborating as a colony to execute a task and the three communication frames that the system has. All modules used in the experimentation had an actuator with 3 rotational degrees of freedom and the axes intersecting at one point. SUPERBOT modular system [5] has also modules with $3 \mathrm{DoF}$, but they are not assembled to work in a wrist-like joint as in this case. Figure 3 shows the actuator and Figure 2 its working area. As it can be observed, the working area is wider than in a 1 or $2 \mathrm{DoF}$ actuator. This characteristic will be translated into an advantage when carrying out manipulation tasks as it will later on be shown. Each module has a high performance DSP where all the control electronics, peripherals, and interfaces are integrated. The design of the module allows accessories to be attached on one end of the module (Figure 10) and they can be detached when not needed (Figure 9). For the experiments carried out and described in this paper two connectors could be distinguished. The

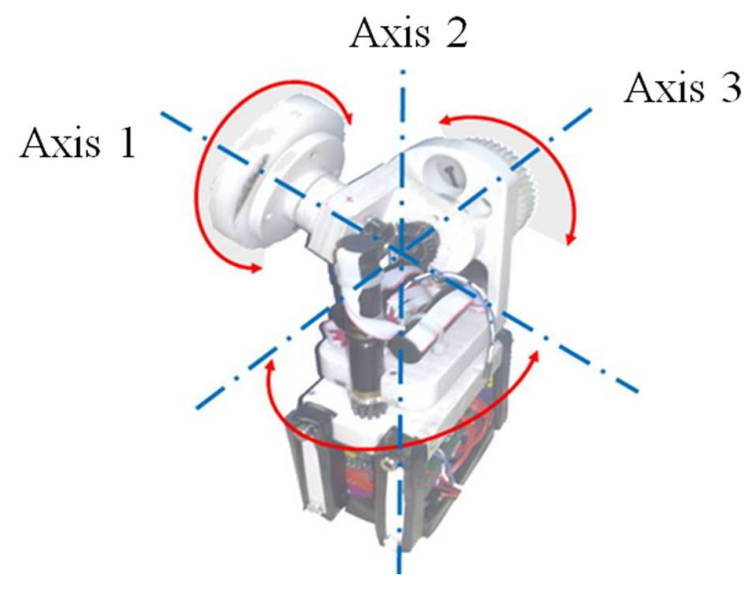

Fig. 3. Mechanical design of the 3-DoF RobMAT module first type, a rigid permanent connector that joined modules in pairs (Figure 4). Modules may or may not be rigidly linked in this way but it was considered necessary for the experiments carried out for reasons of simplicity and better understanding. Let's remark that a module has only 3DoF and what Figure 4(b) shows is two $3 \mathrm{DoF}$ modules joined together, not a $6 \mathrm{DoF}$ module. The other type of connector used is the one that enables reconfigurations and is made out of an electromagnet controlled by the electronics board (male connector) and a metallic plate (female connector), each part embedded on one side of a two module molecule. Figure 5 shows this in detail. The module's dimensions are specified in Figure 6. Its overall weight is $0.785 \mathrm{Kg}$ and it can support a nominal torque of approximately $1.1795 \mathrm{Nm}$, which is somehow more than the torque necessary to lift its on weight (Figure 9.2), with a peak value of $3.5387 \mathrm{Nm}$. Table 1 gives a general overview of RobMAT's system mobility capabilities, DoF and workspace depending on the component considered.

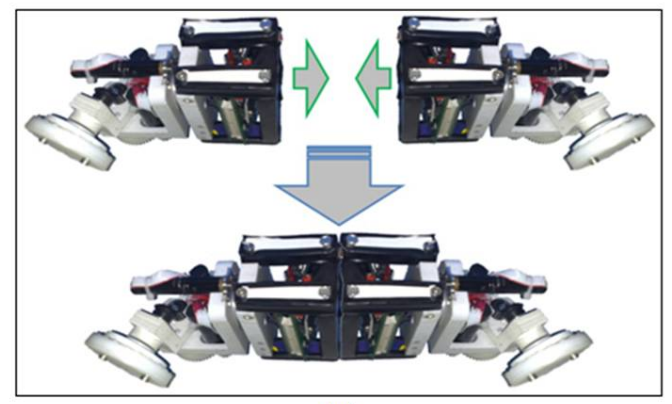

(a)

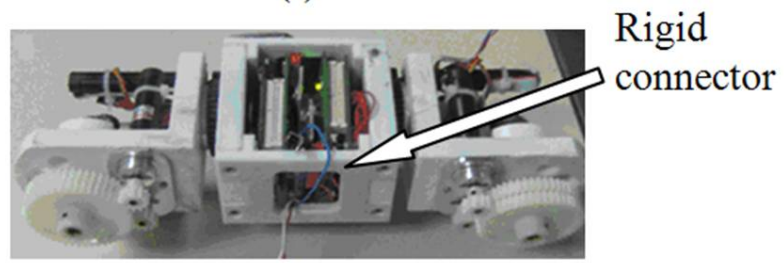

(b)

Fig. 4. (a) Detail of two modules, each with its 3DoF actuator, joined together to form a two module robot via a fixed link. (b) Detail of the rigid connector used for the experiments 
TABLE 1: MAIN FEATURES OF COMPONENTS INVOLVED IN ROBMAT ARCHITECTURE

\begin{tabular}{lclll} 
Component & DOF & Workspace & Communications & Mobility Capabilities \\
\hline $\begin{array}{l}\text { Module } \\
\text { Base Molecule }\end{array}$ & 5 & Sphere surface & CAN bus & Null \\
$\begin{array}{l}\text { (Two modules) } \\
\begin{array}{l}\text { Complex Molecule } \geq 10 \\
\text { (More than two modules) }\end{array}\end{array}$ & $\begin{array}{l}\text { Composition of } \\
\text { some spheres }\end{array}$ & $\begin{array}{c}\text { CAN bus, Bluetooth } \\
\text { and RF }\end{array}$ & Without connectors \\
& & CAN bus and Bluetooth & With connectors or wheels \\
& &
\end{tabular}

\section{EXPERIMENTS AND FIELD WORK}

The execution of complex tasks is generally achieved by the accomplishment of a series of simpler ones where a reconfiguration of the modular system may be at times required. The use of modular robots for motion and telemanipulation offers the advantage that few modules can execute simple tasks and reconfigure with more modules for performing more complicated ones. Below is a description of the experiments carried out using RobMAT's 3DoF modules for performing displacement and manipulation tasks.

\section{A. Displacement: "Wheel” Mode}

Displacement is one of the most needed abilities in the majority of service robots. RobMAT's 3DoF actuator permits two ways of displacement to be carried out depending on the surface the robot is moving ("stepping" or "wheel" mode). The "wheel" mode option is the fastest and most efficient way to displace the modular robot. RobMAT module's mechanical structure has a cylindrical disk on one of its sides and the 3DoF actuator allows this cylindrical disk to work as a wheel being able to rotate forward or backwards along two of the axes of the actuator. Figure 7 shows how to displace over the two possible axes ( $\mathrm{X}$ and $\mathrm{Y}$ ) depending on how the "wheels" are orientated. Beginning from Fig. 7.1, if the right module turns 90 around the y-axis (Fig. 7.2) and the left module -90 around the same axis we get to the configuration shown in Fig. 7.3 where the robot can move along the y-axis.

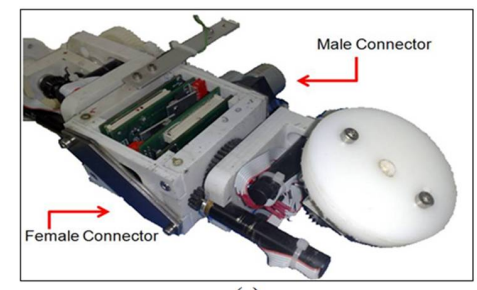

(a)

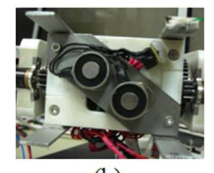

(b)

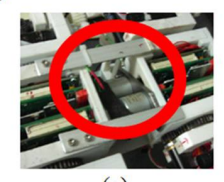

(c)
Fig. 5. (a)Detail and distribution of the electromagnet and plate connector in a two module robot assembly. (b)Male connector based on two electromagnets. (c) Connector detail of two joined base molecules
Again starting from Figure 7.1 if the modules turn 90 and -90 around the $\mathrm{x}$-axis respectively we get the configuration shown in Fig. 7.5 where the robot displaces along the $\mathrm{x}$-axis. With this last configuration it is also possible to change the direction of movement of the robot. Leaving one module still and turning only the other module's "wheel", the robot rotates around a vertical axis that passes through the still module so that it is possible to change the direction of motion any desired angle. This option is recommended for moving over flat or semi-flat surfaces, independently of the type of surface, when speed is important or the robot must move a somehow long distance. With this alternative, equilibrium of the system is not to be worried about because stability is given by the "wheels". The 'wheel' displacement mode allows easy module reconfiguration with the aim of creating complex structures that will accomplish other tasks or are meant to carry out tasks more efficiently. An example of reconfiguration is shown in Figure 8. Two robots consisting of two modules each approach using "wheel movement" and they contact creating a new 4-module structure. Afterwards, and depending on the environment conditions, displacement can be done following the "wheel" mode pattern just explained above or using the "stepping" mode pattern that will be explained in the next section.

\section{B. Displacement: "Stepping” Mode}

When the surface is unstructured, obstacles need to be avoided or when the terrain is made out of some kind of material, substance or liquid that could damage the module's internal parts it is strongly recommended to separate it from the surface which is crossing and displacement using a "wheel" mode wouldn't be appropriate.

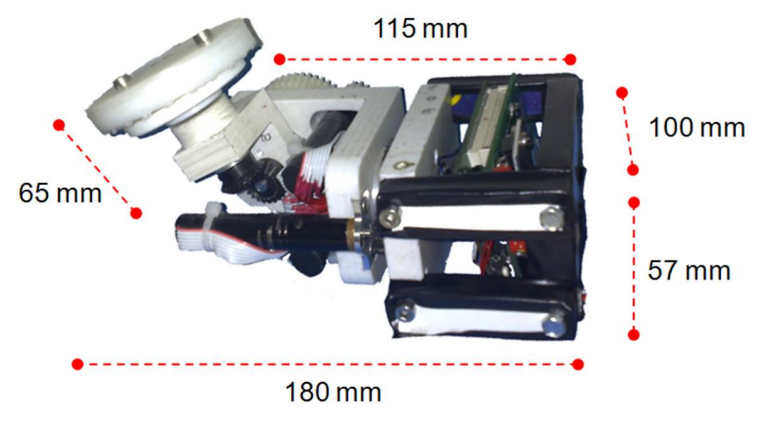

Fig. 6. Dimensions of the module 


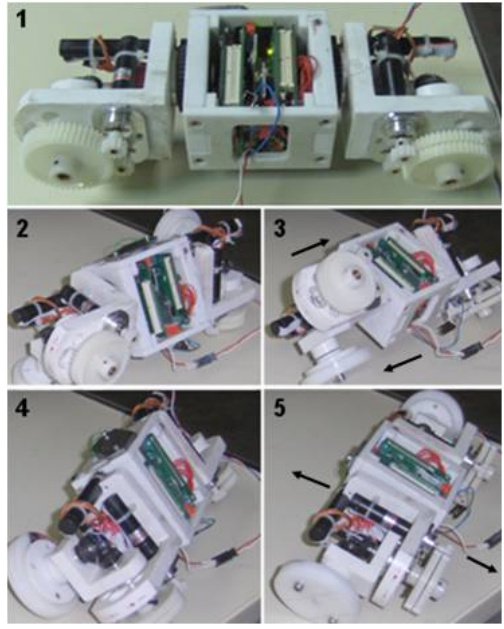

Fig. 7. First kind of movement: rolling over two possible axes depending on the wheels' face orientation

The advantage of having the $3 \mathrm{DoF}$ actuator is that another displacement pattern can be created that will solve the previous problem permitting an efficient displacement to be carried out. When the surface is metallic and the robot has only two modules attached together it is possible to connect an electromagnet to the cylindrical end part of the modules and make it move giving sort of "steps". The procedure requires one of the modules to connect its electromagnet fixing itself to the metallic surface. The actuator then develops enough torque as for lifting the other module and using its $3 \mathrm{DoF}$ actuator rotates and places the lifted module in front of itself, replacing it then over the surface. The electromagnet disconnects and the sequence is repeated with the other module. Figure 9 shows the sequence of movements employed. Some tasks may require the use of tools to be accomplished because of the difficulty of the job, the environment conditions or the precision required.

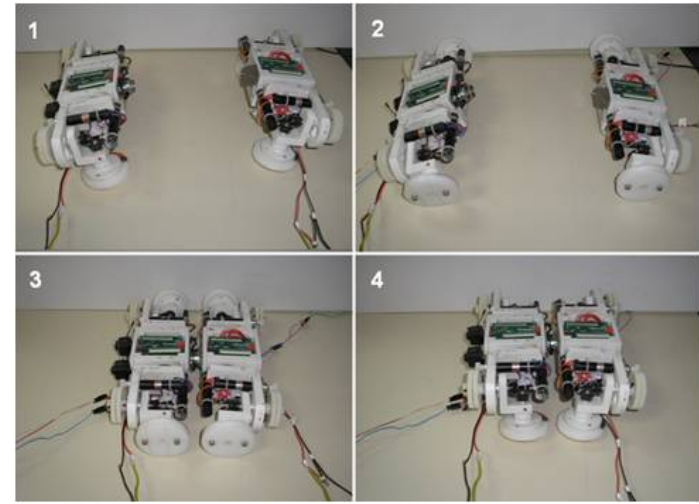

Fig. 8. Reconfiguration into a four module structure

In this sense RobMAT's module gives the possibility to attach an accessory to the module, which was already mentioned in section II. The "stepping" mode just described above can be carried out in the same way with the accessory

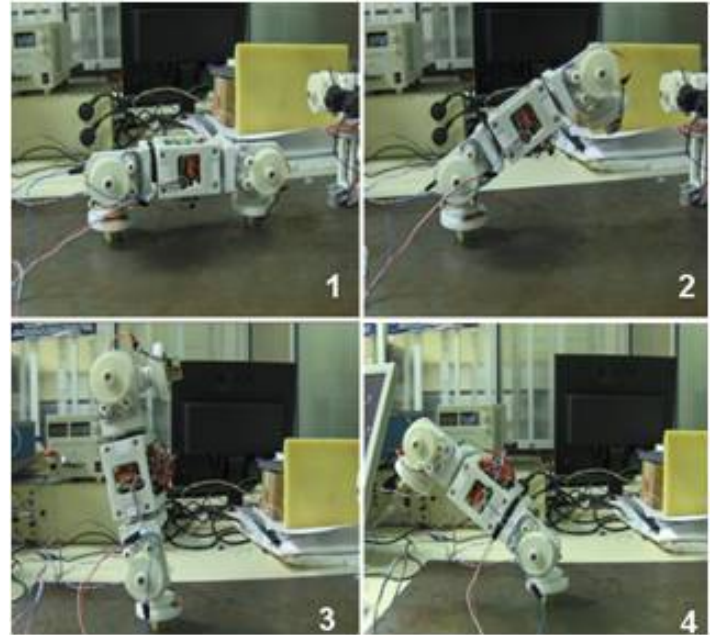

Fig. 9. Displacement sequence for a two module system using electromagnetic devices

attached to the module placing the electromagnet at the end of the accessory. Figure 10 shows this detail. The advantage of attaching the accessory and displacing the robot with it is that the objective of separating the system from the surface it is crossing would be better worked out. Just like in section A modules with an accessory attached can be reconfigured into a four or a six modular system. For both configurations there are displacement step-like patterns, however, the four modules displacement model was shown experimentally to be more efficient because the step turns out to be wider and faster performed. When reconfigured with six modules the central two modules hinder the movements of the other four and it proves itself to be a better configuration for manipulation tasks than for displacement as it will be showed in the next section. Figure 11 shows the step by step sequence of the displacement pattern for the 4 modules system obtained with simulation and in figure 15.3 and 15.4 this pattern is implemented in the real modular system. In the video attached to this paper this displacement mode can be also seen.

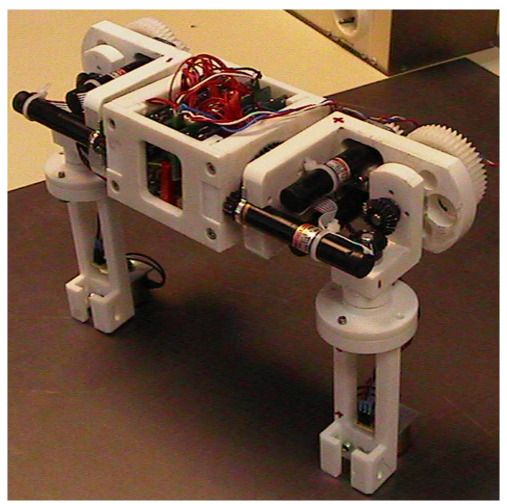

Fig. 10. Two modules with accessory beginning the displacement "stepping" mode pattern 


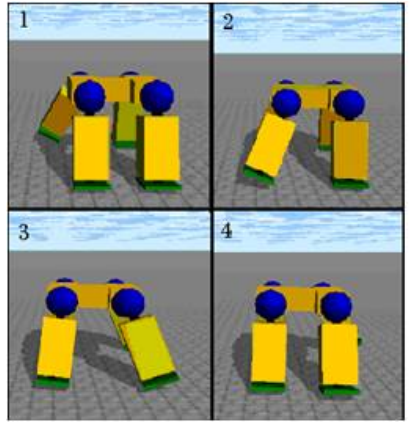

Fig. 11. Simulation of the displacement pattern for a four module with accessory system

Figure 12 illustrates the sequence of movements for the six modules robot displacement. It is observed that for one step, more movements are required in the six modules robot than with the four modules robot and experiments with the real robot modules have shown that the distance advanced in one step is shorter when the robot displaces in the six modules configuration.

\section{Object Manipulation}

When working on metallic surfaces object manipulation can be done as in Figure 13. Two 2-modules robots with accessory attached approach and connect. Once this is done two modules turn on the electromagnets, fix themselves to the surface and lift the other two modules making use of its $3 \mathrm{DoF}$ actuator. This action leaves the other two modules free to manipulate objects. If the surface is not metallic electromagnets cannot be used and it is required to balance the system in some other way so that at least two modules are left free to manipulate objects. With the six module configuration and the $3 \mathrm{DoF}$ motor this is managed using four modules to balance the whole system and leaving the other two to move freely. Because of its wide working area, which was already mentioned at the beginning of the paper, manipulation can be carried out satisfactorily. Figure 14 shows the simulation of a possible distribution.

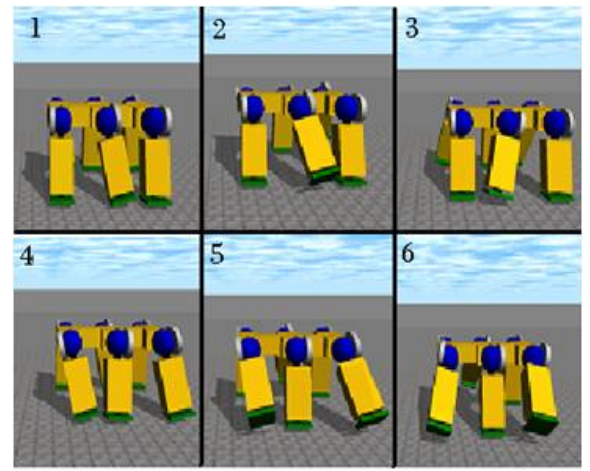

Fig. 12. Simulation of the displacement pattern for a six module robot with accessory

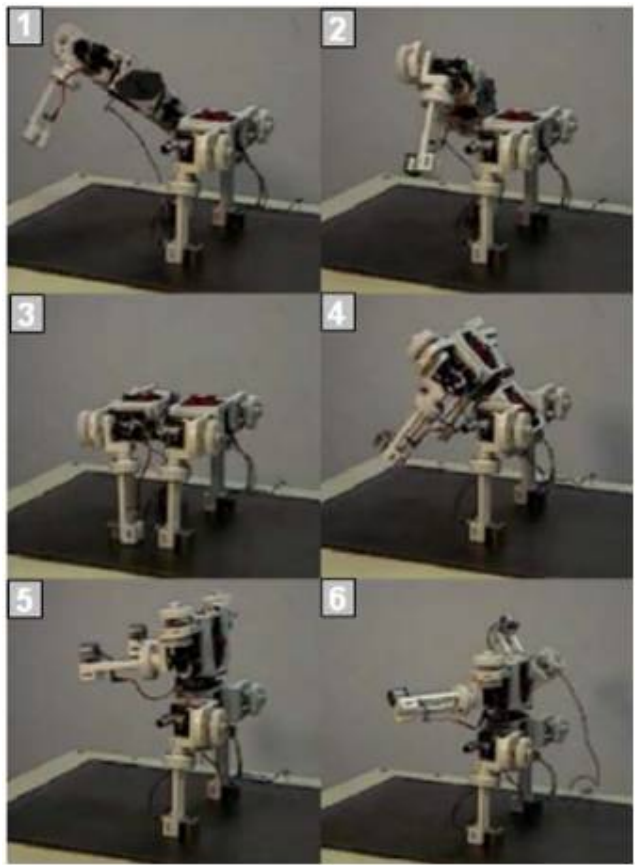

Fig. 13. Approaching and docking sequence for reconfiguration and manipulation task of two molecules

\section{Field Work and Outdoor Movement}

The following experiment shows how using reconfiguration, molecule cooperation and the variety of movements that the $3 \mathrm{DoF}$ actuator offers it is possible to complete complex tasks on field. The test involved three 2-modules systems with the accessory attached. Initially, all three made up a sixmodules robot. With this configuration the robot moved to the working environment that was guided using the images sent by the mini-cameras. Once on site, the two modules carrying the cameras separated and moved to a place where the remote environment could be conveniently perceived.

The remaining modules, configured as a 4-modules robot, moved to the working position where the system reconfigured again into two 2-modules robots that started with the manipulation tasks. Both 2-modules robots where on a metallic surface, where they collaborated in a simple task based on passing a tin plate from one to the other. Figure 15 shows some pictures taken during the experiment.

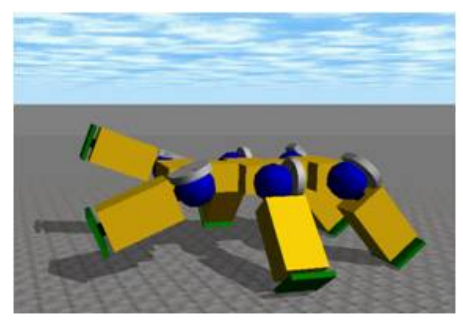

Fig. 14. Configuration with six modules for object manipulation balancing internally the system 


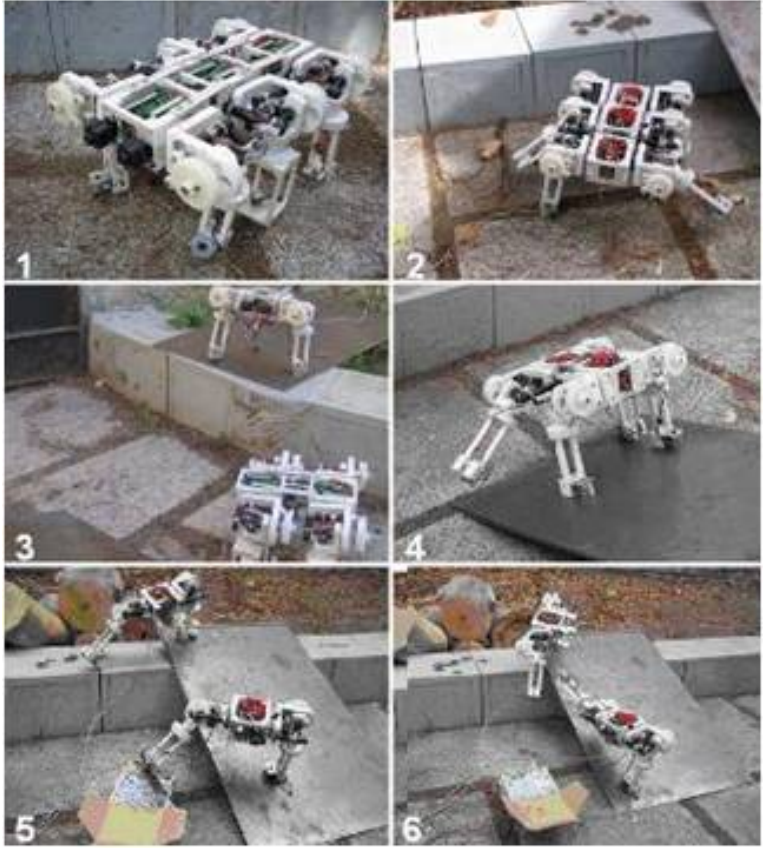

Fig. 15. Field work. Reconfiguration, displacement, manipulation and cooperation tasks developed

\section{CONCLUSION}

It has been demonstrated that a modular robot based on 3 rotational DoF modules is efficient for executing a broad range of tasks. It allows different types of displacement patterns to be developed depending on the surface's characteristics, robot requirements, atmosphere conditions or operation needs. Moreover, it is able to switch from one type to another when desired. It is also important to consider the capabilities that a $3 \mathrm{DoF}$ wrist-like motor can render. Its workspace is wider (a sphere surface) compared to the workspace of 1 or $2 \mathrm{DoF}$ actuator. It has almost no blind spots and is able to reach almost all points with no reconfiguration by moving just one or two modules instead of the entire robot.

Furthermore, the $3 \mathrm{DoF}$ actuator allows the same robot to displace and manipulate without requiring any reconfiguration. Therefore, the task is accomplished more quickly and easily.

Finally, thanks to the versatility offered by the $3 \mathrm{DoF}$ actuator, a complex series of tasks such as those mentioned in "Field work and Outdoor Movement" were carried out. It was done not by using a large number of modules but just six, which is a small number compared to the number of modules that other modular systems with 1 or $2 \mathrm{DoF}$ actuators would need for performing a task sequence of such complexity.

In conclusion, the use of $3 \mathrm{DoF}$ actuator increases the difficulty of control algorithms and coordination patterns of the modular system. However, the advantages offered in carrying out tasks such as, displacement or object manipulation highly justify its application.

\section{ACKNOWLEDGMENTS}

The authors would like to thank CICYT for financing this research project. The RobMAT project has been financed within the Industrial Design and Production Program (DPI2003-00759 and DPI-2006-06493).

\section{REFERENCES}

[1] M. Yim, Y. Zhang, and D.G. Duff, "Modular Robots", IEEE Spectr., vol. 39, no. 2, pp. 30-34, Feb. 2002.

[2] T. Fukuda, S. Nakagawa, Y. Kawauchi, and M. Buss, "Self organizing robots based on cell structures-CEBOT", in Proc. IEEE/RSJ Int. Conf. Intelligent Robots and Systems (IROS), Nov. 1988, pp. 145-150.

[3] S. Murata, E. Yoshida, A. Kamimura, H. Kurokawa, K. Tomita, and S.Kokaji, "M-TRAN: Self-reconfigurable modular robotic system", IEEE/ASME Trans. Mech., vol. 7, no. 4, pp. 431-441. 2002.

[4] M. Shen, B. Salemi, and P. Will, "Hormone-inspired Adaptive Communication and Distributed Control for CONRO Self-reconfigurable Robots", IEEE Trans. Robotics and Automation, vol.18, no. 5, 2002.

[5] B. Salemi, M. Moll, and W.-M. Shen, "SUPERBOT: A deployable, multi-functional, and modular self-reconfigurable robotic system", in Proc. 2006 IEEE/RSJ Intl. Conf. Intelligent Robots Systems, Oct. 2006, pp. 3636-3641.

[6] E.H. Ostergaard, "Distributed control of the ATRON selfreconfigurable robot", Ph.D. thesis, Maersk McKinney Moller Institute for Production Technology, Univ. of Southern Denmark, Nov. 2004.

[7] D. Rus and G.S. Chirikjian, Eds., Autonom. Robots (Special Issue on Modular Robots), vol. 10, no. 1, 2001.

[8] W.-M. Shen and M. Yim, Eds., IEEE/ASME Trans. Mechatron. (Special Issue on Self-Reconfigurable Modular Robots), vol. 7, no 4, 2002.

[9] J. A. Escalera, R. Saltaren, R. Aracil, M. Ferre, and C. Garca, "Base molecule design and simulation of a modular robot robmat", in Proc. 16th IFAC World Congress, Prague. 2005

[10] M. Yim, W. Shen, B Salemi, D. Rus, M Moll, H. Lipson, E. Klavins, G.S. Chirikjian, "Modular Self-reconfigurable Robot Systems - Clallenges and Opportunities for the Future".

[11] CAN, "Technical Specifications", $\quad$ v. 2.0, http://www.wisc.edu/writest/Handbook/DocAPA.html, 1991

[12] BlueTooth, "Bluetooth Consortium Official Web Page", https://www.bluetooth.org/, 2007.

[13] M. Ferre, R. Aracil, and M. Navas, "Stereoscopic Video Images for Telerobotic Applications", Robotic Systems J., vol. 22, no.3, pp. 131146, 2005.

[14] J. A. Escalera, M. Ferre, R. Aracil, and J. Baca, "Teleoperation of a Modular Robot for Collaborative Manipulation", in Proc. 11th KES Int. Conf. Knowledge-Based and Intelligent Information and Engineering Systems, part II, pp. 1204-1213, 2007.

[15] A. Chow, and T. Johnson, "Distributed Operating Systems and Algorithm Analysis. Addison-Wesley", 1997. 